\title{
Music therapy for neonatal stress and pain-music to our ears
}

\author{
Nathalie L. Maitre (ID $^{1} \cdot$ Shmuel Arnon ${ }^{2,3}$ \\ Received: 1 July 2020 / Revised: 31 July 2020 / Accepted: 18 September 2020 / Published online: 30 September 2020 \\ (c) Springer Nature America, Inc. 2020
}

The article by Corrigan et al. in this issue of the Journal of Perinatology [1] highlights the role of music therapy as a support for preterm infants recovering from stressful or painful procedures, in this case, an exam for retinopathy of prematurity. Preterm birth disrupts typical brain maturation with long-term neurodevelopmental consequences [2]. Painful procedures in the neonatal intensive care unit (NICU) can also contribute to maladaptive processing of environmental stimuli and poor social-emotional outcomes [3]. Therefore, nonpharmacological therapeutic and individual developmental care interventions have emerged to mitigate the impact of painful experiences on preterm infants and their caregivers [4]. One such intervention is music therapy (distinct from simple music exposure), a calibrated and individualized approach delivered by highly trained professionals, leveraging musical patterns as scaffolds for physiological and neural responses. Multiple forms of music therapy from cognitive-behavioral to neurological to humanistic-experiential techniques have been trialed in NICUs worldwide [5].

In this issue, Corrigan et al. used a 5-min recording of each child's mother singing a lullaby, mixed with heartbeat, to decrease stress and achieve more rapid recovery after a painful procedure. The choice of mother's voice, the lullaby pattern and range and the volume of the intervention (60 dBA) are all carefully chosen from best evidence in protecting the developing auditory system and using preferred auditory stimuli that can easily be processed and recognized even by those with immature hearing $[2,6]$. The effect of the intervention was measured using the Premature Infant Pain

Nathalie L. Maitre

Nathalie.Maitre@nationwidechildrens.org

1 Department of Pediatrics and Center for Perinatal Research, Nationwide Children's Hospital, Columbus, OH, USA

2 Department of Neonatology, Meir Medical Center, Kfar Saba, Israel

3 Sackler Faculty of Medicine, Tel Aviv University, Tel Aviv, Israel
Profile (PIPP) score by independent masked examiners, and results were compared using rigorous statistical analyses to preterm infants receiving standard care. The authors found that only infants with longer and more painful exams benefited from the music therapy intervention, as demonstrated by greater PIPP score changes. This beneficial effect was not found if the retinopathy of prematurity examination was shorter than that of the mean eye exam $(<83 \mathrm{~s})$. These findings are in agreement with a number of systematic reviews that have demonstrated ambiguous results for the efficacy of various types of auditory stimulation on preterm infants when research populations are clinically heterogeneous $[5,6]$.

While the present study focused on short-term pain and stress relief, other music therapy studies in preterm infants show short-term effects on heart rate variations; even more promising research demonstrates distal effects such as parental well-being, attachment, bonding, anxiety, length of hospitalization, feeding patterns and developmental outcomes [7]. Music therapy stimulation ranges from calm music sung softly or played on an instrument and includes lullabies, improvised music, popular, New Age, classical or family indigenous music. Sometimes the songs or sounds are entrained to infants' vital signs (i.e., synchronized with breathing or heart rate pattern) and sometimes they incorporate womb sounds, heartbeats and parents' voices [8]. Music therapists support parents, nurses, doctors, and other healthcare professionals in providing appropriate auditory stimulation to the infant or even sometimes to the parent [8]. While NICU music therapy studies contribute to a growing area of interest in developmental care [9], this type of developmental care still faces many challenges before it can establish itself as a widespread NICU practice.

At the core of these challenges lay some of the precepts that make music therapy so effective: the almost universal appeal of the sound, rhythm, melody, texture, harmony, structure and form of music. These elements are so intrinsically enmeshed within our auditory experience as children and adults, it is easy to forget that to experience them positively requires complex neural processes that are only emerging in early life $[3,10]$. It can also mislead nonmusic 
therapists into thinking that all auditory stimulation that fits musical patterns is music therapy and beneficial, or that the tuning of musical experience to an infant's neurodevelopmental stage and physiological state is somehow intuitive. In fact, years of training in psychology, music, biological and social sciences, disabilities and general studies are necessary to leverage musical patterns to achieve a therapeutic result [7]. Further complicating matters, some music therapy teachings posit that the music used for therapeutic purposes must be directed to an infant's responses. Unfortunately, training in NICU-specific skills for music therapists is currently offered only in the course of 2-week workshops, insufficient to prepare them for the challenges of understanding the fragility and differences inherent to NICU patients. Most NICU music therapists then acquire their training in NICUs, from other skilled professionals such as occupational and physical therapists. NICU music therapists then face another challenge, common to other developmental care disciplines: the neuroscience of infant sensory systems trails that of children and adults, hindering mechanistically-based designs for auditory interventions and use of objective and relevant outcomes. The links between physiological observations and brain-based processes are often extrapolated from older children or even adults, rather than founded in scientific evidence. This leaves music therapists evaluating the effects of their intervention on imprecise or unrelated metrics. Despite these obstacles, NICU music therapists persevere in their goal of becoming evidence-based health care providers, and music therapy may well offer an attractive complement or alternative to medical treatments when considering the long-term effects of repeated stress and pain on the developing brain [3]. This would be especially relevant if used in combination with proven developmental interventions focused on tactile and proprioceptive pain mitigation along with a family-centered approach.

For any researcher attempting to advance the knowledge base of neonatal developmental care, imperatives of scientific rigor and reproducibility are balanced with support for the well-being of NICU families and prevention of harm in vulnerable patients. As such, for music therapy to be incorporated into broader NICU developmental care, it will need to contribute a body of scientific evidence-base through exactly-designed studies with meaningful outcomes, such as in the Corrigan study. Neonatal teams, patients, families and NICU music therapists can look forward to an exciting new era for this field of research and care.

\section{Compliance with ethical standards}

Conflict of interest The authors declare that they have no conflict of interest.

Publisher's note Springer Nature remains neutral with regard to jurisdictional claims in published maps and institutional affiliations.

\section{References}

1. Corrigan MJ, Keeler JR, Miller HD, Khallouq BA, Fowler SB. Music therapy and retinopathy of prematurity screening: using recorded maternal singing and heartbeat for post exam recovery. J Perinatol. 2020. https://doi.org/10.1038/s41372-020-0719-9.

2. McMahon E, Wintermark P, Lahav A. Auditory brain development in premature infants: the importance of early experience. Ann NY Acad Sci. 2012;1252:17-24.

3. Anderson DE, Patel AD. Infants born preterm, stress, and neurodevelopment in the neonatal intensive care unit: might music have an impact? Develop Med Child Neurol. 2018;60:256-66.

4. Symington A, Pinelli J. Developmental care for promoting development and preventing morbidity in preterm infants. Cochrane Database Syst Rev. 2006;4:38-41.

5. Van der Heijden MJ, Oliai Araghi S, Jeekel J, Reiss IK, Hunink MG, Van Dijk M. Do hospitalized premature infants benefit from music interventions? A systematic review of randomized controlled trials. PLoS ONE. 2016;11:e0161848.

6. Standley J. Music therapy research in the NICU: an updated metaanalysis. Neonatal Netw. 2012;31:311-6.

7. Bieleninik $€$, Ghetti $C$, Gold C. Music therapy for preterm infants and their parents: a meta-analysis. Pediatrics. 2016;138: e20160971.

8. Haslbeck FB. Music therapy for premature infants and their parents: An integrative review. Nord J Music Ther. 2012;21:203-26.

9. Emery L, Hamm EL, Hague K, Chorna OD, Moore-Clingenpeel M, Maitre NL. A randomised controlled trial of protocolised music therapy demonstrates developmental milestone acquisition in hospitalised infants. Acta Paediatrica. 2019;108:828-34.

10. Maitre NL, Key AP, Chorna OD, Slaughter JC, Matusz PJ, Wallace MT, et al. The dual nature of early-life experience on somatosensory processing in the human infant brain. Curr Biol. 2017;27:1048-54. 